Ann. Sci. For., 1988, 45 (4), 341-358

\title{
Etude préliminaire de la variabilité de la pression de sève de clones de Pin sylvestre dans le centre de la France
}

\author{
J. LEVIEUX, H. JACTEL, F. LIEUTIER \\ INRA, Station de Zoologie forestière, Centre de Recherches d'Orléans, \\ Ardon, F 45160 Olivet
}

\begin{abstract}
Summary
Preliminary study of the sap pressure variability between several clones of scots pine in the centre of France
\end{abstract}

To discriminate several categories of resistance of Scots Pine to bark-beetles attacks, a preliminary study of nycthemeral and between clone sap pressure variation was conducted in the centre of France (Nogent-sur-Vernisson, Loiret and Orléans). Measurements were made on several Haguenau and Mossig clones as well as Pines of known maternal genetic stock. Root systems are poorly developed between 0 and $-40 \mathrm{~cm}$. At these depths, the water content of the soil, as indicated by a neutron probe, would lead to a certain drought in summer and an excess of water in winter. Under the climatic conditions of central France, depending on the variations in sap pressure measured, discriminant values could be obtained around 3 p.m (T.U) after a fairly long drought period or 4 a.m when air humidity is highest.

Key words: Sap pressure, clone, exposure, Scots Pine.

\section{Résumé}

Pour définir d'éventuelles catégories de résistance des tiges de Pin sylvestre aux attaques de Scolytes, une étude préliminaire de la variabilité nycthémérale et des fluctuations interclônales de la pression de sève a été effectuée dans le centre de la France à Nogent-sur-Vernisson et à Orléans. Elle a porté sur des clônes Haguenau, Mossig et divers Pins Haguenau d'origine maternelle connue. Leurs systèmes racinaires se développent superficiellement entre 0 et $-40 \mathrm{~cm}$, zone pour laquelle la teneur en eau du sol mesurée à la sonde à neutrons tend vers le dessèchement en été et vers l'engorgement en hiver. Selon les écarts de pression de sève mesurés, on pourrait effectuer des mesures discriminantes de la $P_{s}$ vers 15 h (T.U.) surtout après une période de sécheresse ou vers 3 h (T.U.) lorsque l'humidité atmosphérique est maximale.

Mots clés : Pression de sève, clônes, exposition, Pinus silvestris. 


\section{Introduction}

Les forestiers ont empiriquement établi une corrélation entre le succès des attaques de scolytes lors de leur installation sur les troncs de résineux et l'état physiologique des arbres ayant supporté ou supportant des stress divers induits le plus souvent par une indisponibilité temporaire en eau (Stark, 1965; Berryman, 1972; Hanover, 1975; Cates \& Alexander, 1982). Les pins sylvestres (Pinus silvestris L.) de la Région Centre ayant subi au cours des dix dernières années des dégâts majeurs dus aux Scolytes, dégâts considérablement amplifiés à la suite de la sécheresse marquée de 1976, il nous a paru important de démarrer une étude sur l'état physiologique des arbres pour tenter de préciser les conditions facilitant l'installation des insectes sur les troncs puis l'explosion de leurs populations. De telles connaissances sont indispensables pour mieux cerner les étapes initiales de la démographie des populations de Scolytes.

Si l'on ne peut négliger l'importance des facteurs génétiques, pédologiques ou sylvicoles pouvant intervenir dans l'affaiblissement des pins ni celle de phénomènes biologiques comme les attaques de Diprion pini L. (Hyménoptère Tenthrède) conduisant au même résultat, il paraît peu discutable que l'état hydrique des arbres joue un rôle essentiel. Pour mieux le cerner, nous avons été conduit à étudier la variabilité d'un certain nombre de critères physiques.

On sait qu'en pratique les divers modes d'expression de la résistance naturelle d'une plante sont sujets à controverse (PAINTER, 1951 ; BECK, 1965 ; MAXWELl, 1972 ; Hanover, 1975). Dans le domaine physique, quatre d'entre eux ont fait l'objet de mesures sur diverses essences: la pression osmotique, la teneur en eau du liber, la pression d'exsudation des oléorésines et le potentiel de sève (VITE \& Wood, 1961 ; Wood, 1962 ; Stark, 1965 ; Waring \& Cleary, 1967 ; Chararas, 1970 ; Ritchie \& Hinkley, 1975 ; Raffa \& Berryman, 1982). Nous avons effectué des séries comparatives de mesure des trois derniers de ces critères. Il est vite apparu que les fluctuations de la teneur en eau du liber et de la pression d'exsudation des oléorésines ne fournissaient pas, pour diverses raisons, de résultats utilisables. Aussi ont-ils été écartés et le présent article expose ceux relatifs au potentiel de sève.

\section{Matériel et méthodes}

S'intéresser aux fluctuations des teneurs en eau chez un ligneux, quel que soit le mode d'expression retenu, oblige à connaître aussi la quantité d'eau mise à sa disposition. Nous avons donc mesuré simultanément la pluviosité, la teneur en eau des sols à Nogent à côté des arbres étudiés grâce à une sonde à neutrons et suivi le niveau des nappes perchées avec un réseau de piézomètres.

\subsection{Matériel végétal}

Compte tenu de la complexité génétique du Pin sylvestre et de l'origine souvent inconnue des arbres adultes existant en forêt, nous avons, pour obtenir des résultats comparatifs, mesuré l'ampleur des fluctuations de la pression de sève sur les clônes 
Haguenau 2, 7, 9, 12, 16, 17 plantés il y a 27 ans au domaine des Barres (Nogent-surVernisson, Loiret) et au Centre de Recherches forestières de Nancy-Amance, sur les clônes Mossig 13 à 16 du même âge à Nogent ainsi que sur des Pins âgés de 12 ans existant en double répétition au Centre de Recherches forestières d'Orléans. Il s'agit dans ce dernier cas de descendances Haguenau obtenues à partir de graines issues d'arbres repérés en vergers à graines. On connaît donc l'origine maternelle des tiges sans que l'origine paternelle soit fixée.

Enfin, le système racinaire de quelques tiges a été déterré pour savoir à quelle profondeur s'enfonçaient les racines.

\subsection{Suivi hydrique}

Le parc à clônes de Nogent a été équipé d'un pluviographe implanté sur le site d'expérience entre deux rangées de Pins. Les données obtenues sont comparées à celles relevées par la station météorologique officielle distante de $150 \mathrm{~m}$ environ.

Le sol de Nogent a été étudié dans une fosse pédologique creusée à $30 \mathrm{~m}$ des clônes étudiés.

Les teneurs en eau du sol ont été mesurées hebdomadairement pendant 2 ans à la sonde à neutrons. Le tube de mesure a été implanté entre deux des clônes étudiés. L'abondance des silex dès $-40 \mathrm{~cm}$ a cependant empêché d'enfoncer ce tube en dessous de $-70 \mathrm{~cm}$. Un réseau de piézomètres a été enfoncé jusqu'à la couche imperméable. Il a permis de fixer la position de la nappe superficielle lorsqu'elle existe.

\subsection{La pression de sève}

\subsection{Définition de la pression de sève}

La pression de sève (Ps), mesurée à la chambre à pression de Scholander, constitue une mesure du potentiel hydrique de la plante (Scholander et al., 1965 ; Aussenac \& Chassagne, 1974 ; Kaufman, 1968 ; Aussenac \& Granier, 1978). Les potentiels mesurés sont la résultante à un moment donné de l'approvisionnement et de la demande en eau. Cette intégration reflète ce qui se passe dans la plante d'où son avantage sur les méthodes d'appréciation indirecte à partir des teneurs en eau du sol ou de l'atmosphère. On sait qu'il existe une fluctuation journalière cyclique. Il s'y ajoute, au cours des saisons, une fluctuation liée aux disponibilités en eau du sol (pour une bibliographie détaillée, consulter par exemple ClEARY \& ZAERR, 1980).

\subsection{Méthodes de mesure}

Le principe de la mesure est le suivant:

Le potentiel hydrique $P_{5}$ dans la plante peut être décomposé en

$\mathbf{P}_{\mathrm{s}}=\mathrm{P}_{\mathrm{os}}+\mathbf{P}_{\text {urg }}$ où

$\mathrm{P}_{\mathrm{os}}=$ potentiel osmotique dû aux substances dissoutes

$P_{\text {turg }}=$ potentiel de turgescence des tissus vivants.

La chambre de Scholander permet de mesurer $P_{s}$. Lorsqu'on prélève sur l'arbre une aiguille ou un rameau, la colonne de sève subit brutalement la pression atmosphéri- 
que et se rétracte dans les vaisseaux. En introduisant l'organe coupé dans la chambre, sa section seule restant visible, et en exerçant une pression dans l'enceinte, on note une pression pour laquelle la sève affleure au niveau de la section. Cette pression de sève est égale et de signe opposé au potentiel de la sève avant coupure (d'où le signe devant les valeurs de pression). Le potentiel hydrique ainsi mesuré s'exprime en bar par suite de l'équivalence entre unité d'énergie par unité de volume et unité de pression.

Les mesures ont été effectuées grâce à une chambre alimentée à l'azote sur des rameaux de 1 ou 2 ans. Le délai maximal s'écoulant entre prélèvement et mesure étant de 90 minutes, chaque rameau est placé, pour éviter la transpiration pendant cet intervalle, dans un sac en plastique conservé dans une glacière.

Nous avons étudié la variabilité de divers critères (influence de l'âge du rameau, de son exposition ou de sa hauteur sur l'arbre, etc.) en insistant sur la fluctuation journalière et saisonnière de la pression de sève. L'étude de ces fluctuations du point de vue méthodologique et physiologique a fait l'objet de travaux classiques, dont certains sur le Pin sylvestre (Ritchie \& Hinkley, 1975 ; Hellkvist \& Parsby, 1976). Notre travail ne veut pas en constituer une répétition; il vise seulement à fournir des éléments de comparaison valables sous nos climats, aussi seuls quelques résultats susceptibles d'intéresser l'entomologiste seront-ils décrits dessous.

\section{Résultats}

\subsection{Sols et racines}

Les sols de Nogent sont constitués par un affleurement sablo-limoneux tertiaire de $30 \mathrm{~cm}$ environ d'épaisseur surmontant une argile lourde de structure continue à rognons de silex $^{(*)}$. La présence de cette couche en gênant l'installation correcte du tube a empêché d'effectuer des mesures hydriques valables en dessous de $-70 \mathrm{~cm}$.

Pour examiner l'enracinement, nous avons dessouché à Nogent à l'aide d'une pelle mécanique 2 pins abattus par la tempête. Il n'existe pas de pivots. Les racines d'un diamètre supérieur à $3 \mathrm{~cm}$ partent toutes horizontalement et seules quelques racines minces s'enfoncent dans l'argile. Nous avons aussi examiné 4 autres pins en Forêt d'Orléans abattus par des coups de vent. Les conclusions sont identiques.

Dans tous les cas, on constate une absence de pivot, les racines sont traçantes, enfoncées pour la plupart à moins de $50 \mathrm{~cm}$ et seules quelques radicelles de quelques millimètres de diamètre descendent jusqu'à la nappe. De telles observations confirment les travaux de Beckel (1983) comme ceux de BLOCK (1983) sur l'adaptation racinaire du Pin sylvestre en milieu hydromorphe. On sait par ailleurs que le système racinaire des Pins s'adapte considérablement tant à des conditions très diverses de structure du sol qu'au microrelief (LoRIo et al., 1972).

$\mathrm{Au}$ total, compte tenu d'un enracinement des pins très superficiel limité surtout aux 40 premiers centimètres sous la surface du sol, on peut concevoir que le régime hydrique dans cette couche contrôle la quasi-totalité de l'approvisionnement en eau. Ceci explique l'importance des nappes mais aussi le rôle de la fréquence des pluies sur les niveaux sableux sans réserve et la nécessité de l'étude détaillée du régime hydrique. 


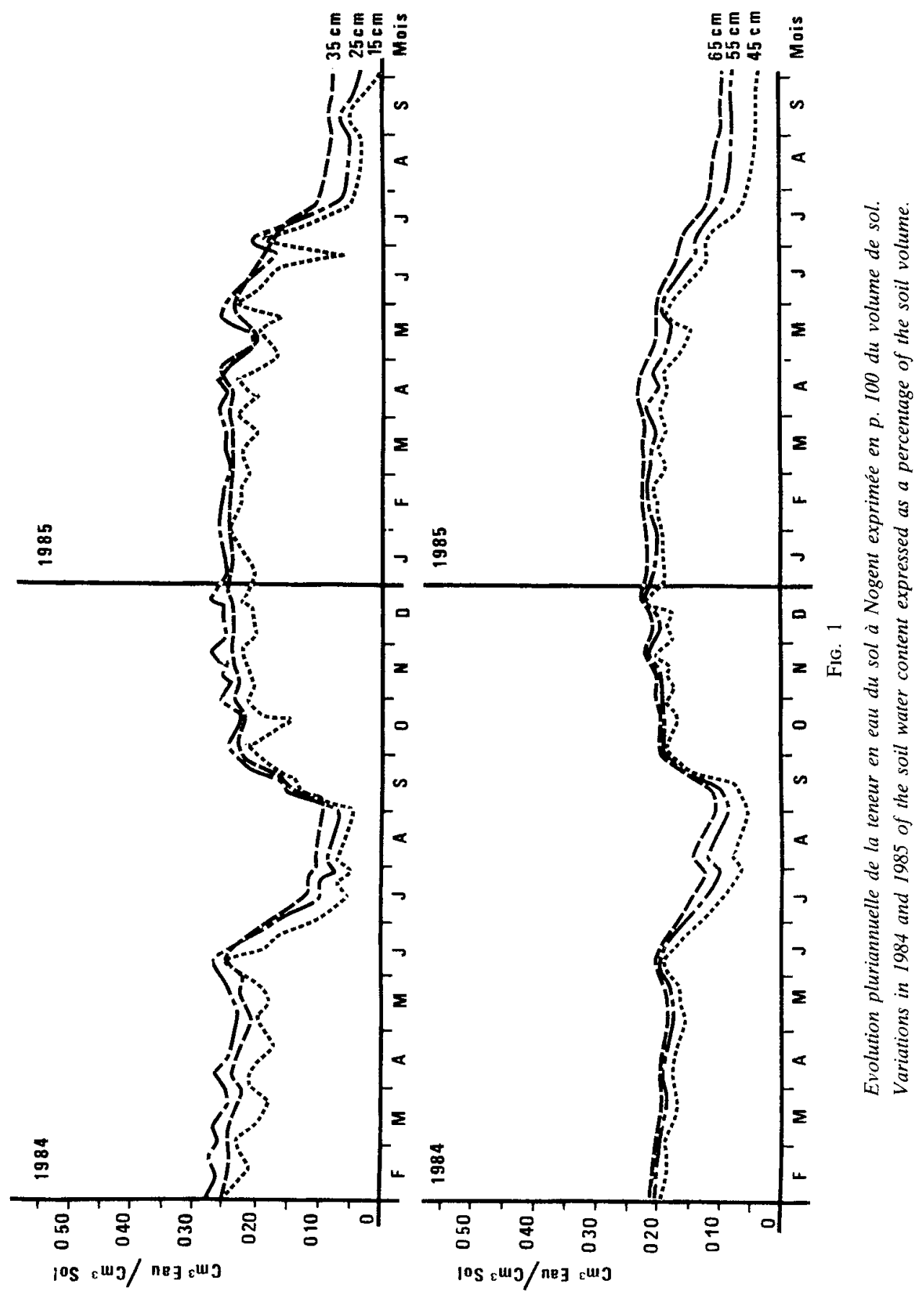




\subsection{Régime hydrique dans les sols}

En 1984 et 1985, il est tombé respectivement 835 et $535 \mathrm{~mm}$ de pluies à Nogent. 807 et 555 en forêt d'Orléans. Si l'écart annuel entre les deux sites paraît négligeable, les variations décadaires entre stations peuvent dépasser $65 \%$.

A Nogent, les arbres sont plantés dans des sols imperméables dès $40-45 \mathrm{~cm}$ de profondeur sur un plateau dont la pente quasi nulle n'autorise guère la circulation latérale de l'eau. Cela conduit à des conditions d'engorgement du sol en surface dès l'automne : en septembre 1984, les précipitations de $150 \mathrm{~mm}$ suffisent à saturer le sol.

En revanche durant les mois d'été, le dessèchement des horizons occupés par les racines est très rapide (fig. 1). Les réserves utiles, estimées à $95-100 \mathrm{~mm}$ ( $R$. DARTHOUT, comm. pers.) sont utilisées à $80 \%$ environ dès le début du mois de juillet 1984 . Au cours des mois de juillet et août, le total des précipitations n'étant que de $58 \mathrm{~mm}$, le dessèchement du sol atteint son maximum à la fin août. La période de sécheresse de 1985, plus tardive mais plus prolongée donne des résultats analogues.

La mauvaise contribution du sol à la fourniture en eau au niveau racinaire peut donc aller jusqu'au stress hydrique lors des années les plus sèches.

\subsection{Variations nycthémérales de la pression de sève}

Pour l'étudier, nous avons prélevé en juin 1985 à Nogent un rameau d'un an, à 3 mètres de hauteur, en lisière nord sur les quatre arbres constituant chaque clône Mossig 13 à 16 , soit 16 mesures par série répétées à 4,10 , 16 et 22 heures en temps universel.

Les courbes de la figure 2 illustrent la variabilité nycthémérale. Il existe un minimum en milieu d'après-midi pour lequel les valeurs de la $P_{s}$ atteignent de -10 à - 13 bars lors de nos mesures et un maximum au milieu de la nuit pour des valeurs de $-4,5$ à $-6,5$ bars. De telles valeurs sont inférieures à celles mesurées en Suède sur Pin sylvestre (Hellkvist \& Parsby, op. cit.). Ceci pourrait être rapproché des conditions météorologiques assez médiocres pour la saison (brume, ensoleillement réduit, température variant de $11^{\circ} \mathrm{C}$ à $4 \mathrm{~h}$ du matin à $17^{\circ}$ à $16 \mathrm{~h} 00$, etc.).

Les variations des valeurs mesurées à une heure donnée entre les 4 tiges d’un même clone sont généralement faibles $(-0,7$ bar entre valeurs extrêmes pour MOS 16 ; $-1,5$ bar chez MOS 15 ; etc.). Les écarts représentent au maximum $20 \%$ de la valeur mesurée.

Seuls les clônes aux performances les plus éloignées semblent pouvoir être séparés (exemple MOS 16 et MOS 15 à 16 heures) (tabl. 1).

L'écart interclônal, insuffisant pour discriminer les clônes entre eux, est maximal en milieu d'après-midi. Peut-être ces écarts sont-ils significatifs vers 3 heures du matin où on atteint le potentiel de base. D'un point de vue entomologique et dans le cas d'une différence de sensibilité des divers clònes aux attaques d'un ravageur, c'est donc vers 15 heures ou 04 heures du matin que ces mesures ont le plus de chance d'être discriminantes. 


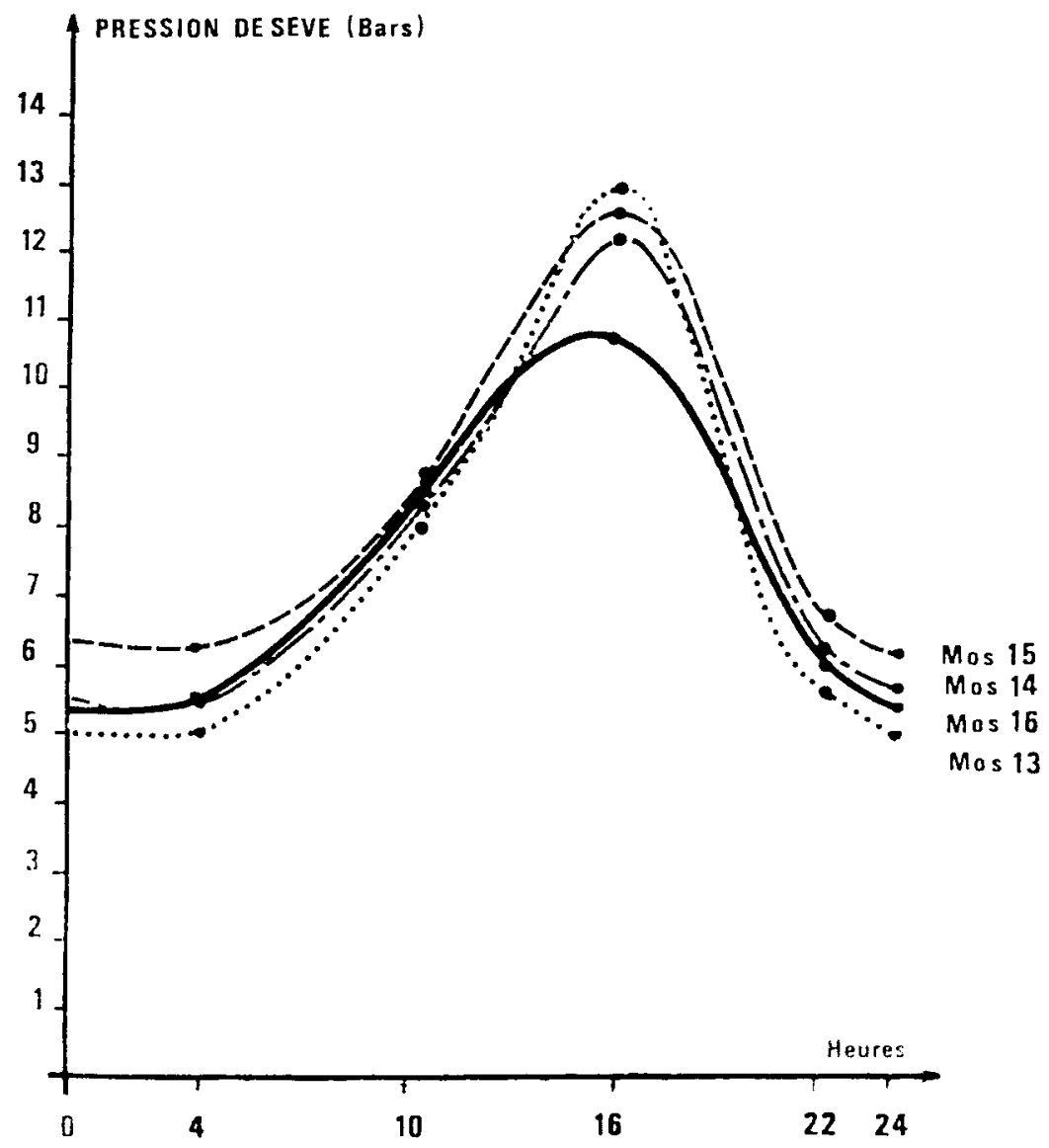

FIG. 2

Variations nycthémérales de la pression de sève mesurée sur 4 clones Mossig de P. Sylvestre (18.05.85 - Nogent-sur-Vernisson).

Diel variations of the sap pressure of 4 Mossig clones of Scots Pine (85.08.15- Nogent-sur-Vernision).

\section{Tabieau 1}

Moyennes et écarts-types des pressions de sève mesurées au cours d'un même cycle nycthéméral sur 4 différents clones Mossig en juin 1985.

Mean and standard errors of the sap pressure measured in 4 trees belonging to 4 different Mossig clones during a diel cucle in June 1985.

\begin{tabular}{|c|c|c|c|c|}
\hline Heure & $16 \mathrm{~h} 00$ & $22 \mathrm{~h} 00$ & 04 h 00 & $10 \mathrm{~h} 00$ \\
\hline$\ldots \ldots \ldots \ldots$ & $-10,68 \pm 0,64$ & $-6.10 \pm 0,36$ & $-5,55 \pm 0.99$ & $-8,33 \pm 0,36$ \\
\hline $\operatorname{MOS} 15$ & $-12.63 \pm 0.75$ & $-6.75 \pm 0.39$ & $-6.25 \pm 0.78$ & $-8.23 \pm 0.41$ \\
\hline $\operatorname{MOS} 14$ & $-12,18 \pm 1,93$ & $-6.23 \pm 0.86$ & $-5.48 \pm 0.59$ & $-7.93 \pm 0.78$ \\
\hline $\operatorname{MOS} 13$ & $-12.53 \pm 1.57$ & $-5,48 \pm 0,49$ & $-5,00 \pm 0,33$ & $-8,10 \pm 0,40$ \\
\hline
\end{tabular}




\subsection{Variabilité inter-clônale}

Pour évaluer la variabilité interclônale, nous avons en pratique mesuré la $\mathrm{P}_{\mathrm{s}}$ chacun des jours de la semaine sur des rameaux $\mathrm{N}+1$ situés à $4 \mathrm{~m}$ de haut coupés à 15 heures, moment où la variabilité de cette pression est supposée être maximale. Cette variabilité a été étudiée sur des échantillons prélevés en juin 1985 sur des descendances Haguenau 2, 7, 9, 12, 16 et 17. Chaque série d'arbres dans chacunes des 4 descendances est représentée par 4 demi-frères disposés par 2 dans 2 situations différentes appelées $\mathrm{N}$ et $\mathrm{N}-\mathrm{W}$. Pour chacun de ces arbres, 2 échantillons ont été prélevés, un au nord, l'autre au sud.

Le tableau 2 montre que, à une même date, l'écart type représentant la variabilité intradescendance est toujours supérieur ou égal à la différence entre les moyennes représentant la variabilité interdescendance.

TABLEAU 2

Etude de la variabilité de la pression intra-provenance entre 6 descendances "Haguenau" de Pins Sylvestres (rameaux âgés de 2 ans, hauteur: $1,40 \mathrm{~m}, 16$ heures).

Intra-provenance variability of the sap pressure between 6 different Haguenau progenies of Scots Pine (shoots : 2 years old, Height: $1.40 \mathrm{~m}, 04.00$ p.m.).

\begin{tabular}{|c|c|c|c|}
\hline \multirow{2}{*}{ Provenance } & \multirow{2}{*}{ Date } & \multicolumn{2}{|c|}{ Pression de sève (bars) } \\
\hline & & Moyenne & Ecart-type \\
\hline $\begin{array}{r}7 \\
9 \\
12 \\
17\end{array}$ & 17.06 .85 & $\begin{array}{l}-10,6 \\
-10,1 \\
-10,5 \\
-11,0\end{array}$ & $\begin{array}{l}0,6 \\
0,8 \\
1,0 \\
0,8\end{array}$ \\
\hline $\begin{array}{r}9 \\
12 \\
17\end{array}$ & 18.06 .85 & $\begin{array}{l}-12,2 \\
-12,0 \\
-12,1\end{array}$ & $\begin{array}{l}1,8 \\
0,7 \\
0,3\end{array}$ \\
\hline $\begin{array}{r}7 \\
12\end{array}$ & 19.06 .85 & $\begin{array}{l}-\quad 3,7 \\
-\quad 4,3\end{array}$ & $\begin{array}{l}0,4 \\
0,6\end{array}$ \\
\hline $\begin{array}{l}7 \\
9\end{array}$ & 20.06 .85 & $\begin{array}{l}-11,0 \\
-11,1\end{array}$ & $\begin{array}{l}0,7 \\
0,9\end{array}$ \\
\hline $\begin{array}{l}12 \\
17\end{array}$ & 21.06 .85 & $\begin{array}{l}-\quad 6,7 \\
-\quad 7,7\end{array}$ & $\begin{array}{l}0,5 \\
2,1\end{array}$ \\
\hline $\begin{array}{r}2 \\
9 \\
12\end{array}$ & 2.09 .85 & $\begin{array}{l}-16,2 \\
-16,0 \\
-15,4\end{array}$ & $\begin{array}{l}0,9 \\
1,1 \\
0,6\end{array}$ \\
\hline $\begin{array}{r}2 \\
12 \\
16\end{array}$ & 3.09 .85 & $\begin{array}{l}-13,6 \\
-12,3 \\
-12,7\end{array}$ & $\begin{array}{l}1,0 \\
0,5 \\
0,8\end{array}$ \\
\hline $\begin{array}{r}2 \\
9 \\
16\end{array}$ & 4.09 .85 & $\begin{array}{l}-15,6 \\
-15,2 \\
-14,9\end{array}$ & $\begin{array}{l}0,1 \\
1,3 \\
1,8\end{array}$ \\
\hline $\begin{array}{r}9 \\
12 \\
16\end{array}$ & 6.09 .85 & $\begin{array}{l}-14,3 \\
-13,9 \\
-13,9\end{array}$ & $\begin{array}{l}0,4 \\
0,1 \\
0,6\end{array}$ \\
\hline
\end{tabular}




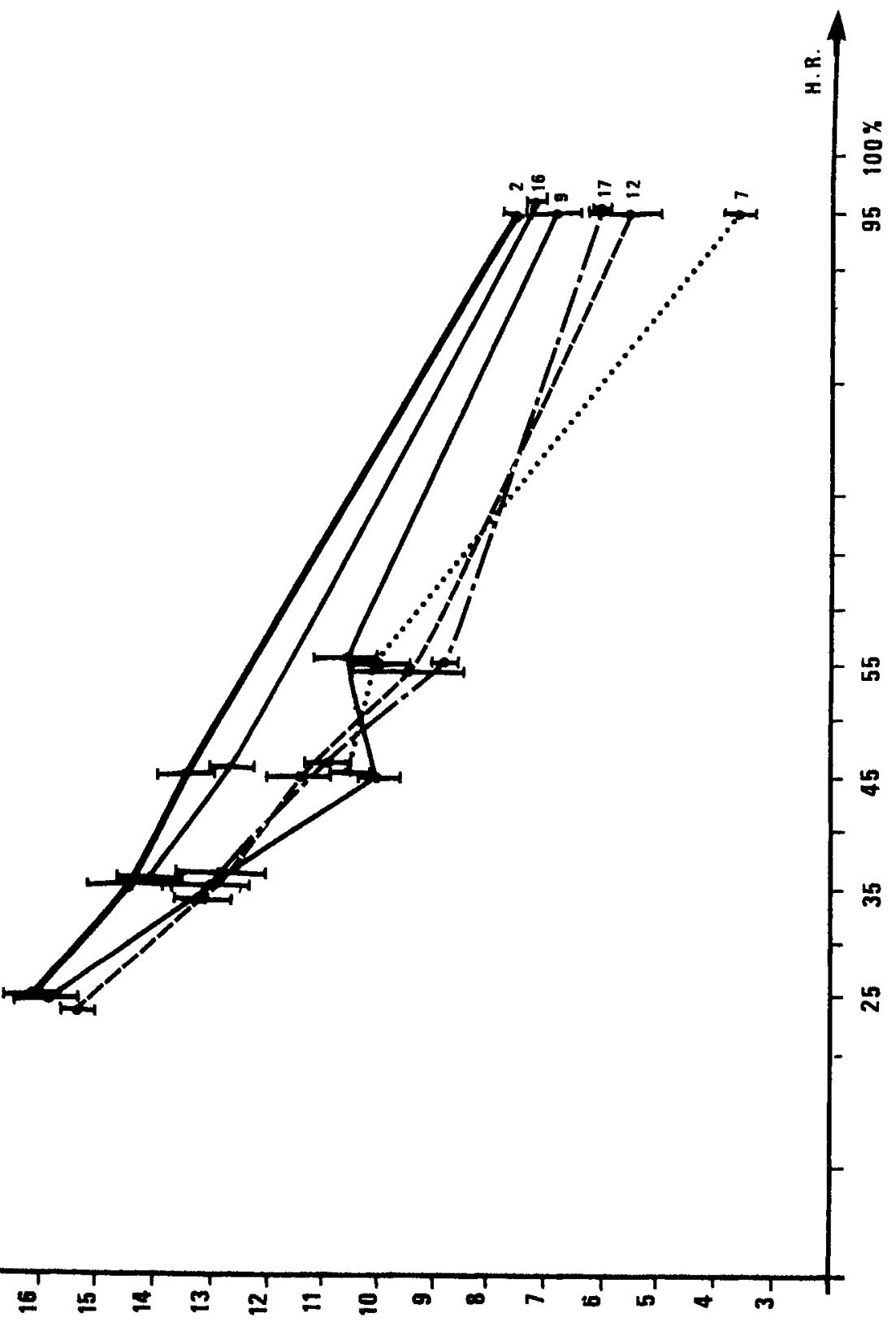

FIG. 3

Représentation graphique des écarts de $P_{s}$ mesurés sur 6 clónes Haguenau en fonction de l'humidité relative de l'air.

Values and standards-errors of the sap pressure of 6 Hagueneau progenies of Scots Pine in relation to the relative humidity of the air. 
Pour séparer au mieux les clones, nous avons reporté les moyennes de $\mathbf{P}_{\mathrm{s}}$ mesurés sur 6 clones Haguenau $(2,7,9,12,16,17)$ en fonction de l'humidité relative de l'air relevée aux heures de mesure (fig. 3 ).

On note ici la forte corrélation négative Ps-humidité de l'air. On constate que certaines valeurs s'écartent significativement les unes des autres (Hag 7 d'Hag 12. Hag 12 d'Hag 9) pour les pressions mesurées lorsque l'H.R. est proche de la saturation.

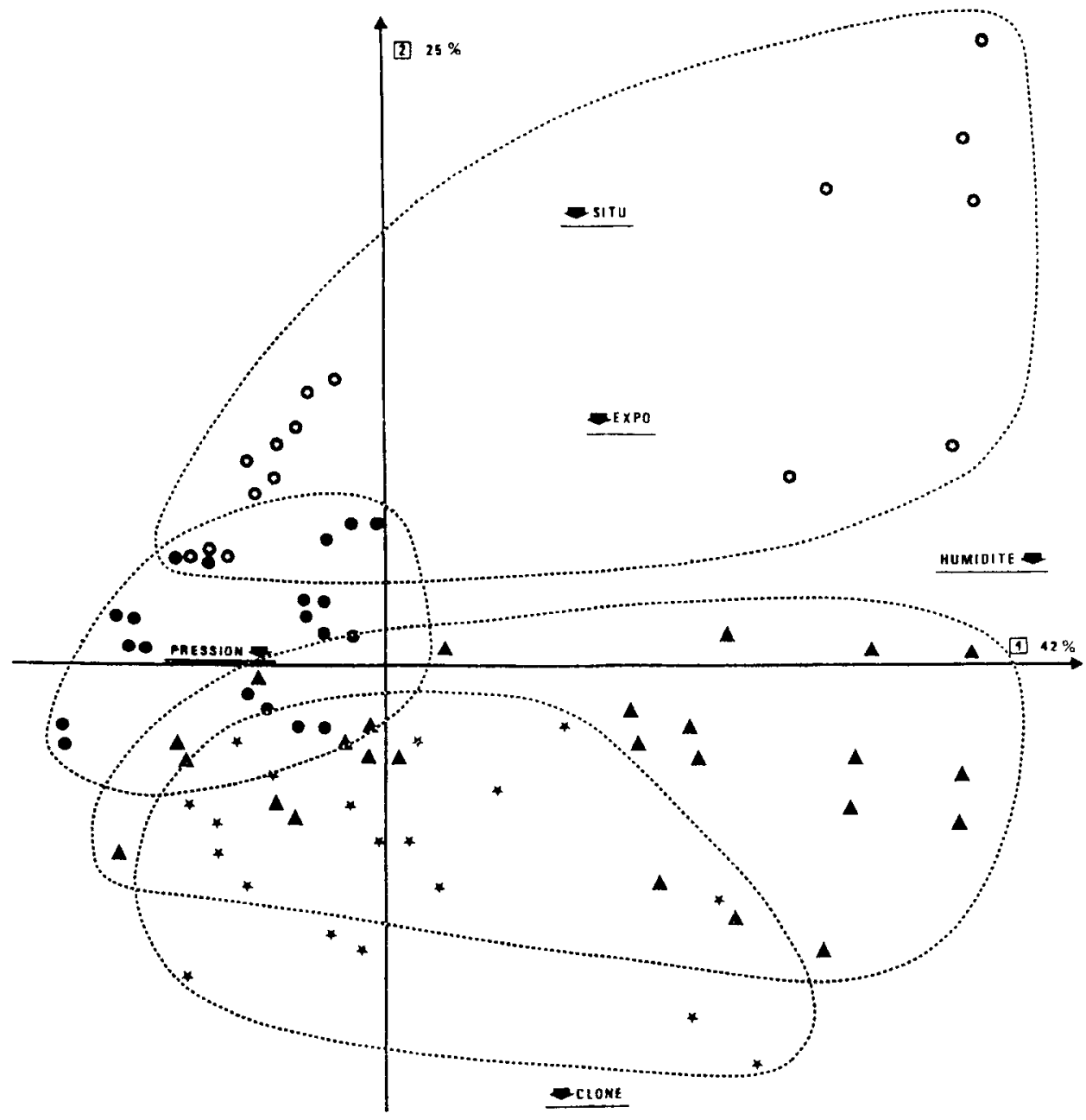

FIG. 4

$I^{r e}$ analyse - Répartition des clônes selon les axes pression de sève humidité ( $A X E$ 1) et situation-exposition ( $A X E$ 2).

First principal components analysis. Distribution of the clones in relation to the components $I$ (sap pressure - humidity) and 2 (situation - localisation).

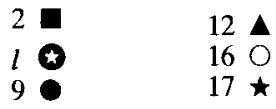


Pour mieux séparer le poids relatif des divers critères nous avons procédé à une analyse factorielle des correspondances (A.F.C.) portant sur les échantillons des clones $7,9,12$ et 17 . Les variables suivantes ont été analysées : clône ou provenance, situation dans la parcelle, exposition du rameau prélevé, hygrométrie et pression de sève. Nous avons ainsi effectué le même travail sur 76 échantillons issus des Haguenau 2, 9, 12 et 16 en septembre 1985 en tenant compte des critères suivants : situation, hygrométrie et pression de sève. Enfin, une analyse de variance portant sur les variables «pression de sève ", "hygrométrie ", " situation » et "provenance » des 76 échantillons de la $2^{\mathrm{e}}$ série a été réalisée.

Les résultats de la première A.F.C. montrent un certain recouvrement entre nuages de points (fig. 4). Ceci semble indiquer que la variabilité intraclônale est plus grande ou égale à la variabilité interclônale pour la pression.

Les valeurs utilisées dans la deuxième A.F.C. ont été traitées deux fois. Dans le $2^{\mathrm{e}}$ traitement (fig. 5 et 6 ) on n'a retenu que les données significatives.

On peut tirer les résultats suivants :

a) Sur le premier axe, il existe une nette corrélation négative humidité pression. Ce résultat paraît logique lorsque i'on considère que la pression de sève exprime un potentiel hydrique. Remarquons aussi que les provenances sont les mieux séparées, écart-type compris, pour les fortes valeurs d'humidité. Celles-ci sont voisines des valeurs nocturnes où l'arbre atteint son potentiel de base (pression de sève minimum).

b) Les nuages de points représentant les descendances ne se succèdent pas le long de l'axe humidité-pression. La pression ne peut donc expliquer la différence entre les provenances, c'est-à-dire, en pratique, servir à les discriminer.

Dans le deuxième système d'axes, il n'existe pas de succession des nuages de points le long de l'axe situation. La variable ne permet pas non plus de discriminer les provenances pour leur $\mathrm{P}_{\mathrm{s}}$; il ne semble pas y avoir de corrélation entre situation et pression.

c) En considérant certains points de la $2^{\mathrm{e}}$ analyse avec les seules données significatives, on peut considérer que les points représentant les données relevées en conditions sèches indiquent une succession de clones le long de l'axe pression. De même les points représentant les données relevées en conditions très humides tendant à indiquer eux aussi une certaine succession (Points reliés en traits pleins sur la figure 6).

L'analyse de variance montre enfin que seule la pression de sève est significativement corrélée avec l'hygrométrie. Les facteurs "descendance " et «situation" ne modifient pas cette corrélation. Cette conclusion rejoint celles précédemment énoncées.

En pratique, il semblerait exister dans quelques cas une discrimination possible des clones par la pression. En effet, on pourrait utiliser les fortes valeurs de pression vers 15 heures après une phase de sécheresse la plus prononcée possible. On pourrait aussi utiliser les périodes de forte humidité où les arbres sont alors proches de leur potentiel de base. Il conviendrait alors de procéder aux mesures vers 4 heures du matin. 


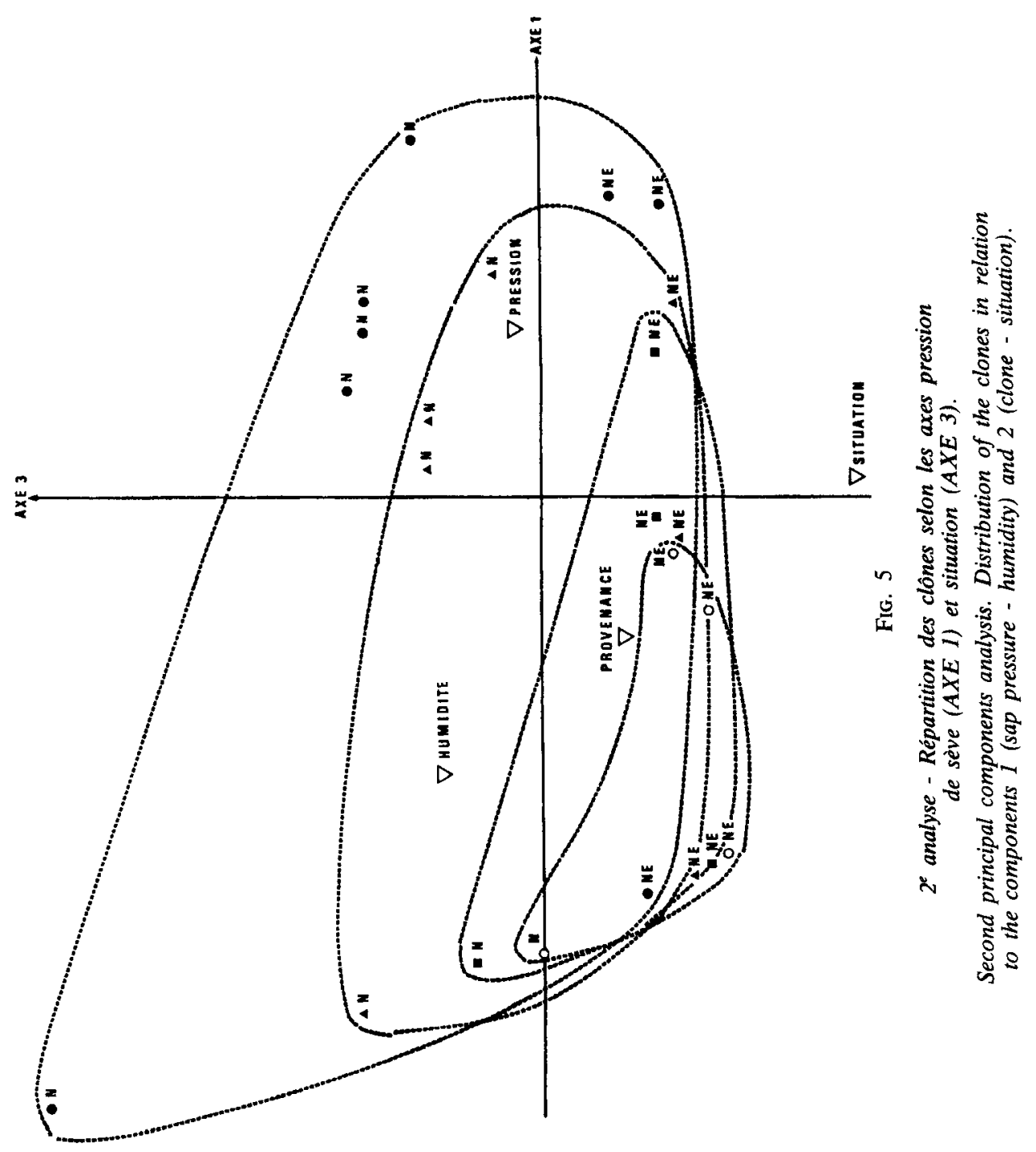




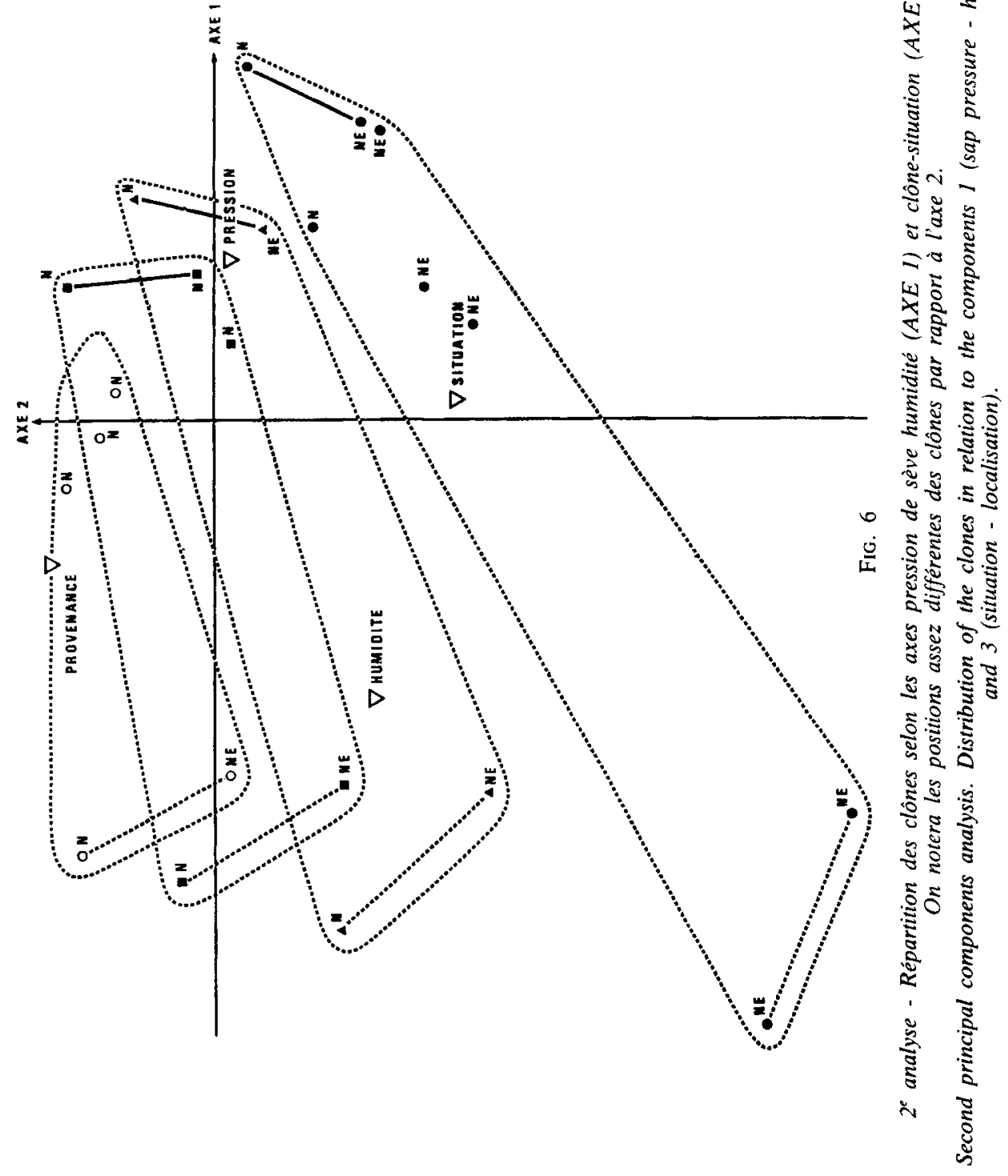


En l'absence de stress hydrique marqué, les mesures de pression de sève mettent en évidence une amplitude de la variation journalière de l'ordre de -10 bars dans les conditions météorologiques moyennes de l'Orléanais ; l'étendue de cette variance est un peu inférieure à celle mesurée en Suède. Les fluctuations interclônales de la variation nycthémérale de la $\mathrm{P}_{\mathrm{s}}$ ne dépassent pas $20 \mathrm{p}$. 100 des valeurs mesurées.

Pour obtenir des valeurs discriminantes de la pression de sève entre tiges, on peut en pratique se limiter à une mesure journalière vers 15 heures sur des rameaux $N+1$ d'exposition identique; il serait souhaitable que ces mesures aient lieu après une période de sécheresse assez longue. On peut aussi, dans une certaine mesure, tenir compte des valeurs nocturnes relevées vers 4 heures du matin lorsque l'humidité ambiante est maximale. Le critère pression de sève pourrait alors, à côté de critères structuraux regroupés en indices comme celui de Waring \& PITMan (1980) par exemple, aider à séparer les tiges d'un peuplement en quelques catégories de résistance.

Deux séries de mesures complémentaires pourraient être envisagées dans l'avenir. La première consisterait, à l'issue d'une longue période sèche, à refaire les mêmes opérations pour déterminer l'ampleur des écarts extrêmes des valeurs. Dans la seconde on mesurerait les valeurs relevées sur divers clônes soumis à des infestations expérimentalement contrôlées de Scolytes pour relier ce critère au degré de sensibilité aux attaques.

\section{Remerciements}

Les auteurs adressent leurs vifs remerciements à Madame Duval et Monsieur Darthout pour la réalisation des études de sol et régime hydrique (INRA-SESCPF, Orléans) et Messieurs Aussenac \& Granier pour leur aide et leurs suggestions.

Reçu le 2 février 1987.

Accepté le 19 janvier 1988.

\section{Références bibliographiques}

Aussenac G. et Chassagne L., 1974. Rapport préliminaire sur la mesure du potentiel de sève dans le rameau et les feuilles. Publ. int. Station Sylviculture et production, $8 \mathrm{p}$.

Aussenac G., Granier A., 1978. Quelques résultats de cinétique journalière du potentiel de sève chez les arbres forestiers. Ann. Sc. forest., 35 (1), 19-32.

Beck S.D., 1965. Resistance of plants to insects. Ann. Rev. Entomol, 10, 207-232.

Beckel J., 1983. Autoécologie des différentes espèces résineuses en milieu hydromorphe. Etudes des adaptations racinaires. Mém. E.N.I.T.E.F., 86 p.

Berryman A.A., 1972. Resistance of Conifers to invasion by bark-beetle fungus associations. Bioscience, 22, 598-602.

BLOCK J., 1983. Ermittung der Wurzelftiefe von Kiefern und Stieleichen zur Beurtcilung der möglichen Ausswirkungen von Grundwasserabsenkungen auf den Wald. In : Root colology and 
its pratical applications. A contribution to the investigation of the whole plant. IRDING, Austria, Bundesanstalt Gumpenstein, 567-576.

Cates R.G., Alexander H., 1982. Host resistance and susceptibility. In "Bark beetles in North American conifers ». Mitron J.B. \& Sturgeon K.B. Edit., Univ. of California press., 527 p.

Chararas C., 1970. Ecologie des Scolytidae. Bull. Soc. Ecol, 3,-169-188.

Cleary B.D., Zaerr J.B., 1980. Water status and carbon dioxyde exchange studies. Part C. : Pressure chamber techniques for monitoring and evaluating seedling water status. New. Zealand J. of For. Science, 10, (1), 133-141.

Hanover J.W., 1975. Physiology of tree resistance to insects. Ann. Rev. Entomol., 20, 75-95.

Hellkvist J.. Parsby J., 1976. The water relations of Pinus silvestris III Diurnal and seasonal patterns of water potential. Physiol. Plant., 38, 61-68.

KaUfman M.R., 1968. Evaluation of the pressure chamber technique for estimating plant water potential in forest tree species. For. Sci., 14, 369-374.

Lorio P.L., Howe V.K., Martin C.N., 1972. Loblolly pine rooting varies with microrelief on wet sites. Ecol., $53(6), 1134-1140$.

Maxwell F., 1972. Host plant resistance to insect nutritional and pest management relationships. In "Insects and mites nutrition", J. Rogriguez, ed. North Holland Publ. Company, Amsterdam, 559-609.

PAINTER R.H., 1951. Insect resistance in crop plants. Univ. Press of Kansas, Lawrence.

Raffa K.R. et Berryman A., 1982. Physiological differences between lodgepole Pines resistant and susceptible to the mountain pine beetle and associated micro-organisms. Environ. Entomol., 11 (2), 486-492.

Richtie G.A., Hinckley T.M., 1975. The pressure chamber as an instrument for ecological research. Adv. Ecol. Res., 9, 165-254.

Scholander P.F., Hammel H.T., Bradstreet E.D., Hemmingsen E.A., 1965. Sap pressure in vascular plants. Science, 148, 339-346.

StaRk R.W., 1965. Recent trends in forest entomology. Ann. Rev. Entomol, 10, 303-324.

ViTE J.P., WoOD D.L., 1961. A study on the applicability of the measurement of oleoresin exudation pressure in determining susceptibility of second growth Ponderosa Pine to bark beetle infestation. Contrib. Boyce Thompson Inst., 21, 67-78.

Waring R.H., Cleary B.D., 1967. Planty moisture stress : evaluation by pressure bomb. Science, 155, $1248-1254$.

Waring R.H., Pitman G.B., 1980. A simple model of host resistance to bark beetle attack. For. Res. Lab., Orcgon State Univ., Corvallis, Res. Note 65.

Wood D.L., 1962. Experiments on the interrelationships between oleoresin exudation pressure in Pinus ponderosa and attack by Ips confusus (L.C.). Can. Entomol., 94, 473-477. 